\title{
Imaging Bioprinted Model Systems for Metastatic Breast Cancer Studies
}

Jessica Riesterer, Arun Singh, Erin Stempinski, Steven Adamou, Cecilia Bueno, Hannah Smith, Claudia Lopez and Joe Gray

Oregon Health and Science University, Portland, Oregon, United States

The SMMART ${ }^{\circledR}$ clinical trials being conducted at OHSU have identified ultrastructure in cancer cells that could serve as potential therapeutic targets. ${ }^{1}$ These ultrastructural features include, but are not limited to, macropinosomes, altered nuclear structure, filopodia-like protrusions, and mitochondrion density. Model systems can now be developed to reproduce these observations and test treatment hypotheses. Customized three-dimensional bioprinted material is one such model. Each bioprint can be tailored to a specific cell population in order to reproduce tumor composition and heterogeneity. In addition, bioprints are 3D structures which more closely represent in vivo cell behaviors than traditional 2D plated cell cultures.

All bioprints were processed and imaged using identical methods. Samples were placed in hard fixative (Karnovsky's fixative solution) $^{2}$ directly from the printer. A modified Hua protocol was used to post-fix and resin embedded samples for imaging. ${ }^{3,4}$ The FEI Helios NanoLab G3 DualBeam ${ }^{\mathrm{TM}}$ focused ion beam-scanning electron microscope (FIB-SEM) was used to collect large-format 2D images over a large area of resinembedded samples at $4 \mathrm{~nm} /$ pixel resolution using the Maps ${ }^{\mathrm{TM}}$ software package. Bioprints were imaged in 3D once the models were optimized using either FIB-SEM or serial block face-scanning electron microscopy (SBF-SEM). This 3D data was collected on either the previously mentioned Helios G3 DualBeam, the Zeiss Crossbeam 550 FIB-SEM, or the Apreo Volumescope 2 SBF-SEM platform depending on the amount of volume and resolution desired. High-resolution data for ultrastructural viewing was collected via FIB-SEM, while heterogeneity throughout the bioprint as a whole was captured via SBF-SEM.

An Organovo, Inc. 3D bioprinter was used to produce preliminary prints of two different types: "cancer pocket" and "no cancer pocket." The initial goal for the prints was to test compatibility with EM imaging. Post-fixation staining and resin-embedding can be harsh to cells grown in the laboratory. Typically, cells are pelleted to create a dense mass to withstand the multiple washes during the processing procedure. Depending on composition, bioprints are not as dense as cell pellets and run the risk of disintegrating during processing. In this case, all bioprints were successfully preserved. However, the workflow must be carefully completed to prevent dehydration during transfer from the printer to fixation. Large open vesicles within cells have been observed in early bioprints and indicate a loss of ultrastructure from poor fixation. Placing the prints into fixative solution as quickly as possible improves this and prevents the lacy structure of remnant cells due to dehydration.

Despite artifacts initially, both types of bioprint show promise for future use as a model system. The "cancer pocket" prints were comprised of an outside stroma containing a mix of $75 \%$ human mammary fibroblasts (HMFs) and 25\% human mammary endothelial cells (HMVECs). A cancer cell pocket existed within the outside stromal component and contained a mix of 75\% MCF7 cells and 25\% HMVECs. The final structure contains approximately 1-1.5 million cells, with an overall composition mix of 10/30/60\% MCF7/HMVEC/HMF cells. The interface between the two compositions is retained and easily viewed at low magnification (Figure 1a). Presumably MCF7 cells, found in the center cancer pocket region show several ultrastructural features found in the human cancer tissues. As seen in Figure 1b, cells have fenestrated nuclei, invaginated nucleoli, and long filopodia-like protrusions reaching into the extracellular matrix. All three of these features are potentially therapeutic targets in metastatic breast cancer.

The "no cancer pocket" prints were quite different. In this case, only one cell mixture was used, consisting of 20/60/20\% MCF7/HMVEC/HMF (total cell percentage). However, the MCF7 cells were in the form of precultured microspheroids $\sim 10,000$ cells in size. Prints still consisted of 1-1.5 million cells in total. Clear 
organization of different cell populations were observed over hundreds of micrometers, mimicking tumor heterogeneity in patient samples. Similar cancer-associated ultrastructure was also observed at a cellular scale. Representative 2D images are shown in Figure 2. ${ }^{5}$
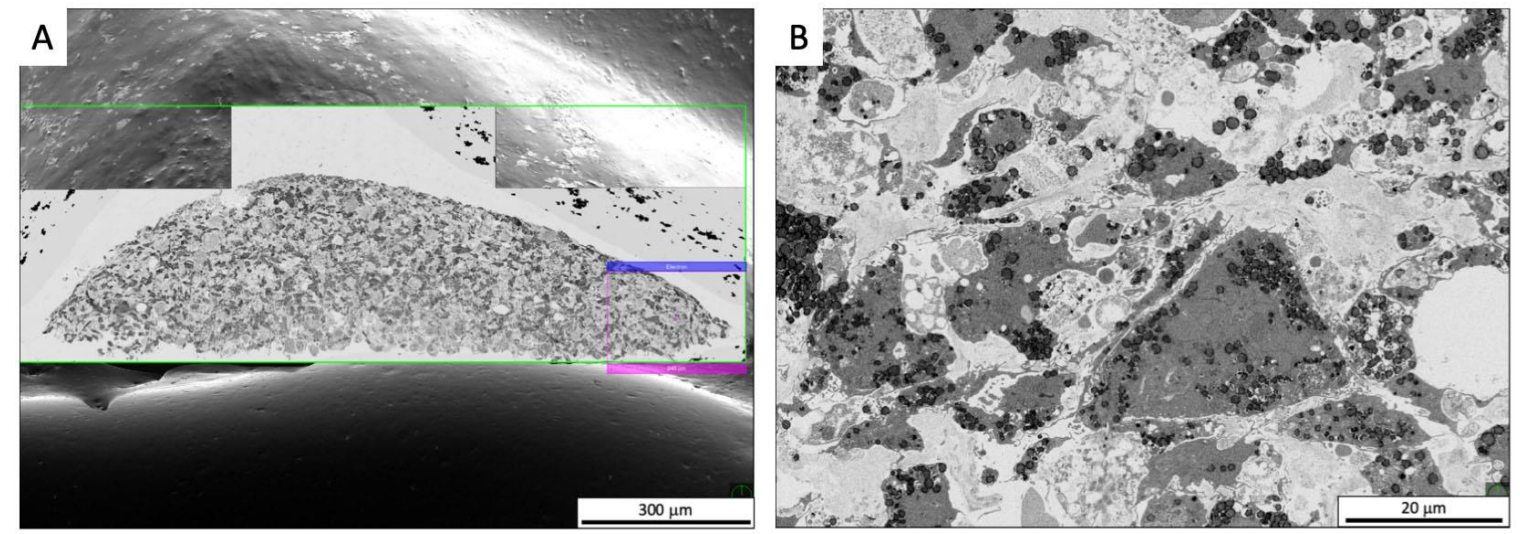

Figure 1. a) A bioprint comprised of a cancer pocket and an outside stromal component shows an interface between the two at low magnification. b) At higher magnification, cells within the cancer pocket show cellular ultrastructure that matches what is observed in human cancer, including long filopodia reaching into the ECM.
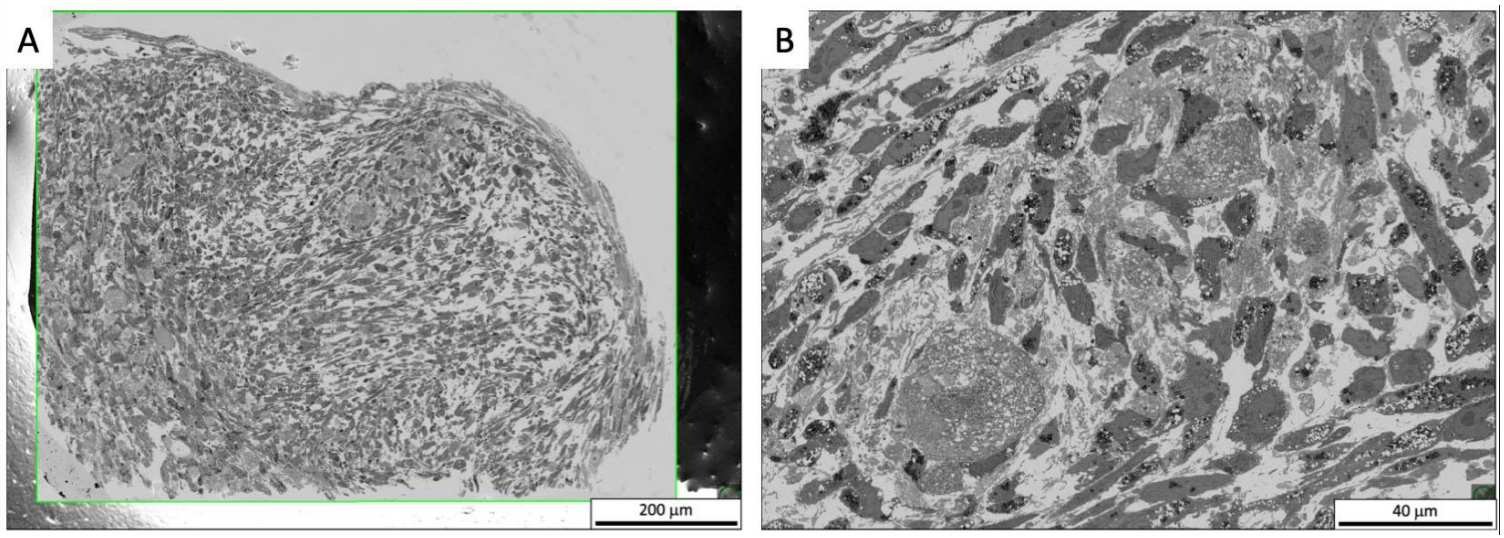

Figure 2. a) Large-scale heterogeneity of cell type populations are clearly maintained and mimic human tumors, while b) cellular ultrastructure at higher magnification shows finer detail of the organization.

\section{References}

1. Johnson, B. et al. Abstract 3296: SMMART: Serial measurements of molecular and architectural responses to therapy. Cancer Research 78, 3296 (2018).

2. Karnovsky, M.J. A Formaldehyde-Glutaraldehyde Fixative of High Osmolality for Use in Electron Microscopy. Journal of Cell Biology 27, 137-8A (1965).

3. Hua, Y., Laserstein, P. \& Helmstaedter, M. Large-volume en-bloc staining for electron microscopy-based connectomics. Nature Communications 6, 1-7 (2015).

4. Riesterer, J.L. et al. A workflow for visualizing human cancer biopsies using large-format electron microscopy. bioRxiv, 675371 (2019).

5. The authors wish to acknowledge the OHSU Multiscale Microscopy Core for instrument access. Funding was graciously provided by the OHSU Center for Spatial Systems, OHSU University Shared Resources, and the NCI Cancer Systems Biology Measuring, Modeling, and Controlling Heterogeneity (M2CH) Center awarded to Joe Gray (5U54CA2099880). 